\title{
Genetics of Obesity in Asia
}

\author{
Eva Maria Cutiongco-de la Paz \\ Director \\ Institute of Human Genetics, National Institutes of Health \\ University of the Philippines, Manila
}

\section{Introduction}

The obesity epidemic is recognized globally as a significant public health concern. The World Health Organization estimates that approximately 2-3 billion people will be overweight and over 700 million will be obese by $2015 .{ }^{1}$ Earlier identified as a problem confined in developed countries, many developing countries have recently shown a significant rise of obesity cases. ${ }^{2}$ It is a major cause of disability and premature deaths in both developed and developing countries from obesity-related diseases such as cardiovascular disease, strokes and complications of diabetes.

\section{Epidemiology of Obesity in Asia}

The prevalence rates of obesity are reported to be very high in North America and Europe with much lower rates in Asia. ${ }^{3}$ However, it has been recently demonstrated that the number of obese individuals in most Asian countries has increased dramatically. ${ }^{2}$ Yoon et al in 2006 reported survey figures from 1993-2001 demonstrating that Thailand had the highest rate of obesity in Asia at $6.8 \%$ among adults being obese followed by Singapore at $6 \%$; China, Taiwan and Malaysia had prevalence figures between 4 and $4.4 \%$ of obese adults. ${ }^{4}$ Hong Kong had 3.8\% of obese adults followed by the Philippines at 3.3\%, Korea at $3.2 \%$ and Japan at $3.0 \%$ India had the lowest percentage of obese adults at $2.2 \%{ }^{4}$

\section{Genetics and Obesity}

Although socioeconomic and lifestyle changes are major contributing factors in the development of obesity, it has been proven in many studies that genetics also play a significant role. Obesity is regarded as a multifactorial condition wherein susceptibility is determined by the interplay of genetics and environmental factors. ${ }^{5}$ It is generally regarded as a complex genetic disorder. Like many other common chronic conditions such as hypertension, diabetes and cancer, obesity usually has an onset in adulthood, demonstrates familial aggregation and

ISSN 0857-1074

Copyright (C) 2011 by the JAFES

Received October 17, 2011. Accepted October 18, 2011. does not show Mendelian patterns of inheritance ${ }^{6}$. Discovering genetic risk factors for complex disorders such as obesity can pave the way for the development of molecular-based susceptibility testing for prediction of disease (presymptomatic testing) ${ }^{6}$.

The contribution of genetic factors to obesity has been well established through twin and family studies. Studying sets of twins, who are reared together or apart allows one to determine whether a condition has a strong genetic influence over the environment and vice versa. The heritability of fat mass seen in twin studies was $40-70 \%$ while a concordance rate of 0.7-0.9 between monozygotic twins compared to 0.35-0.45 among dizygotic twins has been observed. ${ }^{8}$ These studies have proven that heritable factors are responsible for as much as $45-80 \%$ of variations observed in body mass index among individuals and risk of obesity while heritability of obesity in families have been reported between 20 and 50\%.5,7

In the three billion base pairs of the human genome, variations exist on the average, every 500 to 1000 base pairs. These stable, heritable variations in the genome are called polymorphisms and are present in greater than $1 \%$ of the population. Some of these genetic polymorphisms are functionally significant and can have effects on the gene product. They can be used for disease association studies and can act as markers for disease susceptibility. ${ }^{9}$ One of the most widely studied variations are called single nucleotide polymorphisms (SNPs) where a nucleotide base is replaced by another nucleotide base.

\section{Methods of Identifying Genes Associated with Obesity}

A number of research tools are now available to study these variations in the genome that may confer an increased susceptibility to obesity among individuals in different populations. These genetic approaches commonly used to identify the genes related to obesity include candidate gene and genome-wide association studies. Candidate gene studies find an association between variants in an individual's genome within or very

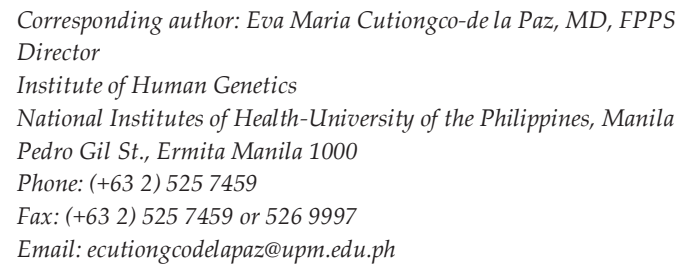


near candidate genes and a disease of interest such as obesity. There is a priori knowledge of the biological function of the candidate gene to be tested because of its involvement in regulating the development of the disease being evaluated. ${ }^{5}$ Genome-wide association studies on the other hand, involves scanning many markers randomly throughout the genome to find genetic variations associated with a disease. However, there is no a priori hypothesis about any particular gene or genes of interest, and its greatest advantage is the discovery of novel genes along biologic pathways with no apparent relation to the pathophysiology of obesity. Genetic studies have repeatedly demonstrated that some genes are associated at a higher frequency among the obese than in the non-obese individuals suggesting that a considerable proportion of weight variation may be due to genetic factors.

It is reported that there are approximately 127 genes associated with obesity from candidate gene association studies. ${ }^{5}$ These genes chosen for their role in various metabolic processes have been replicated in many studies worldwide. The genes that have been identified so far are involved in a variety of biological processes that include regulating food intake or how food is efficiently or inefficiently metabolized in the body and subsequently stored as fat. The association of variations found in a number of these genes and risk for obesity will be described in some studies done among different Asian populations.

\section{Studies on Genetics of Obesity amongst Asians}

The evaluation of genetic susceptibility loci for obesity has been extensively carried out in the Chinese population. Shi $\mathrm{J}$ et al validated five loci from previous genome-wide association studies, obesity-related phenotypes in adult Chinese women. These include FTO, MC4R, BAT2, $S E C 16 B$, and SH2B1. The FTO gene is the fat mass and obesity associated gene and is also known as alphaketoglutarate-dependent dioxygenase enzyme. It is widely expressed in different tissues with the highest concentration in the hypothalamus and pancreatic cells. The $M C 4 R$, on the other hand, is the gene that encodes the melanocortin 4 receptor. Both FTO and MC4R genes have been consistently reported in different populations to be associated with obesity. ${ }^{10} M C 4 R$ has been recognized to play a significant role regulation of food intake and energy homeostasis. ${ }^{5}$ BAT 2 is the human leukocyte antigen $\mathrm{B}$ (HLAB)-associated transcript 2, a gene highly expressed in the hypothalamus. The other candidate genes were SEC16 homolog B (Saccharomyces cerevisiae) (SEC16B) and SH2B adaptor protein 1 ( $S H 2 B 1$ ). It has been suggested through observations that neuronal $S H 2 B 1$ protein regulates energy balance and glucose homeostasis, possibly by enhancing hypothalamic leptin signalling. ${ }^{10}$ However, the mechanism linking the BAT2 and SEC16B genes to obesity is still unknown. The calculated odds ratios for obesity in these different genes ranged from 1.46 (95\% confidence interval (CI): 1.12, 1.92) for BAT2 to 2.16 (95\% CI: 1.39, 3.37) for $M C 4 R .^{10}$ Interestingly, the authors further examined a genetic risk score, calculated by summing the number of risk-increasing alleles that each woman carried at these 5 loci, and they found a significant association with the prevalence of obesity. Women carrying 5 or more risk alleles had a 3.13-fold (95\% CI: 2.06, 4.77) higher prevalence of obesity than women carrying 1 or no risk. ${ }^{10}$

Another population-based study involving a cohort of 3,210 Chinese Hans conducted by Qi et al, investigated the prohormone convertase (PCSK1) gene and predisposition to obesity and type 2 diabetes. This gene encodes an enzyme that converts inactive prohormones such as proinsulin, proglucagon and proopiomelanocortin into biologically active peptide hormones. ${ }^{11}$ Mutations in this PCSK1 lead to impaired glucose tolerance and common variants in this gene have been reported to be associated with obesity among Europeans. The authors sought to replicate these previous studies to determine if the same variants of the PCSK1 gene might predispose to obesity among the Chinese. They were only able to demonstrate modest evidence for association of the PCSK1 variant rs6234 with BMI and overweight in men only and not among women and concluded that it may not be an important contributor to obesity in the Chinese population. ${ }^{11}$

Because several independent studies have reported strong associations of variations in the fat mass and obesityassociated (FTO) gene with obesity among Europeans, Chang Y-C et al in 2008 did replication studies on the role of these FTO gene variants in obesity and type 2 diabetes in the Chinese population. They genotyped 19 single nucleotide polymorphisms of the FTO gene and analyzed its association with obesity in 638 cases and 1610 controls. They were able to confirm an association of FTO genetic polymorphisms with obesity and BMI in the Chinese population with an effect size on obesity risk and BMI comparable with previously reported risk among the Europeans. $^{12}$

A similar study on FTO variants and association with obesity was done among the Chinese and Malay population in Singapore. Tan J et al investigated the association between 9 previously reported FTO SNPs with obesity, type 2 diabetes and related traits in participants of the 1998 Singapore National Health Survey which included 2,919 Chinese, 785 Malays, and 594 Asian Indians as well as 2,996 Malays recruited from another study. The investigators found that variants at the FTO gene are associated with obesity in ethnic Chinese and Malays living in Singapore. ${ }^{13}$

Likewise, a study on the variations of the FTO gene and its association with severe obesity has been conducted among the Japanese by Hotta K et al in 2008. They investigated 15 SNPs on the FTO gene using 927 cases of adults with 
obesity and 1,527 normal-weight controls. A significant association in intron 1 SNP rs1558902 with severe obesity was found among the Japanese with an odds ratio (OR) adjusted for age and gender of 1.41 [95\% confidential interval $(\mathrm{CI})=1.22-1.62 ; \mathrm{p}$ value of 0.0000041$]{ }^{14}$

Genome-wide association studies have shown that variations in the insulin-induced gene 2 (INSIG2) are associated with obesity; such association has been seen in Caucasian populations. ${ }^{15}$ INSIG2 is a ubiquitously expressed gene known to be downregulated by insulin in the liver, involved in fatty acid synthesis and an intermediate in the feedback control of cholesterol synthesis. ${ }^{15}$ Hotta $\mathrm{K}$ and his colleagues investigated the relationship of the INSIG2 gene SNP rs7566605 and obesity among the Japanese from a total of 908 severely obese cases and 1,495 controls. The results of this study showed that the rs7566605 SNP of the INSIG2 gene was significantly associated with obesity with an odds ratio of 1.61 [95\% confidential interval $(\mathrm{CI})=1.24-2.09]$ adjusted for gender and age and may therefore influence the risk of severe obesity in the Japanese population. ${ }^{16}$

Cha S. et al (2009) similarly examined the effect of the rs7566605 polymorphism near the INSIG2 gene on both obesity- and cholesterol-related traits among 2,364 Koreans. However, they failed to demonstrate any association of the rs7566605 polymorphism with cholesterol- and obesity-related phenotypes, although there was a suggestion of the possible involvement of INSIG2 with the plasma level of the total cholesterol in women included in this study. ${ }^{17}$

In a study done among Filipinos from Cebu by Marvelle A. et al in 2008, the researchers found a significant association between the FTO gene and susceptibility to obesity. ${ }^{18}$ This conclusion was drawn from data gathered from 1,886 adult Filipino women from the Cebu Longitudinal Health and Nutrition Survey (CLHNS) cohort. The researchers investigated the association of obesity related traits and 19 SNPs previously identified in 10 genes namely $A D R B 2, A D R B 3, F T O$, GNB3, INSIG2, $L E P R, P P A R G, T N F, U C P 2$, and UCP3 that had been previously reported to be associated with an obesityrelated quantitative trait. The other candidate genes included in this study, apart from those previously described in the other studies above, are the ADRB2 and $A D R B 3$ genes, which are adrenergic beta 2 and beta 3 receptors with the latter being involved in the regulation of lipolysis and thermogenesis. ${ }^{5}$ GNB3 codes for guaninenucleotide-binding protein ( $\mathrm{G}$ protein) and together with $A D R B 2$ and $A D R B 3$ are genes which are part of the catecholaminergic pathway. $L E P R$ codes for the leptin receptor and is a member of the class 1 cytokine receptor family and thought to be involved in the regulation of body weight. PPAR-G, PPARG, the peroxisome proliferator-activated receptor gamma, is a nuclear receptor which regulates fatty acid storage as well as glucose metabolism. The uncoupling proteins UCP2 and UCP3 function to uncouple ATP production from mitochondrial respiration with an end result of affecting the efficiency of energy metabolism. ${ }^{19}$ This candidate gene study done in a cohort of Filipinos was able to show the association of the A allele of FTO variant rs9939609 with BMI and waist circumference. They also demonstrated evidence of association with the homozygous $\mathrm{T}$ allele of ADRB3 rs4994 with BMI, waist circumference and percent body fat; however, only the rs4994 SNP reached statistical significance, not observed in previous studies. The authors concluded that a SNP within the first intron of FTO was associated with BMI among this cohort of Filipino women from Cebu and as also seen in other population groups, the gene maybe important in susceptibility to obesity. ${ }^{18}$

\section{Conclusions}

Knowing the genes related to obesity have far reaching effects individually and globally. Genetic information can be used to predict an individual's predisposition to the development of obesity and its complications. It also allows for the discovery of novel and personalized treatment strategies to be applied to different population groups being cognizant of the fact that inter-ethnic differences exist. The genetic and genomic studies being done among Asian populations will contribute greatly to the wealth of information on genetic predisposition to obesity. These unique and innovative genomic approaches and discoveries may lead to a better understanding of molecular pathways that give rise to obesity and can pave the way to better management and control of obesity, not only in Asia but throughout the world.

\section{References}

1. World Health Organization. Obesity and overweight. Fact Sheet No. 311, September 2006.

2. Ramachandran A and Snehalatha C. Rising burden of obesity in Asia. Journal of Obesity. 2010.

3. Nguyen D and Serag H. Epidemiology of obesity. Gastroenterol Clin North Am. 2010; 39(1): 1-7.

4. Yoon K-H, Lee J-H, Kim et al Epidemic obesity and type 2 diabetes in Asia. The Lancet 2006;368; (9548): 1681-1688.

5. Vimaleswaran K and Loos R. Progress in genetics of obesity. Expert Rev Mol Med. 2010; Feb 26;12e7

6. Motulsky A/ J Zhejiang Genetics of complex diseases.SCIENCE B 2006 7(2):167-168.

7. Farooqi IS and O'Rahilly S Genetic factors in human obesity. Obesity Reviews.2007;8 (Suppl 1) 37-40.

8. Stunkard AJ, Foch TT, Hrubec Z A twin study of human obesity. JAMA. 1986;256:51-54.

9. Daly AK and Day CP Candidate gene case-control association studies: Advantages and potential pitfalls. Br J Clin Pharmacol.2001; 52:489-499.

10. Shi J et al. Evaluation of genetic susceptibility loci for obesity in Chinese women. Am J Epidemiol. 2010;172:244-254

11. Qi Q et al. Association of PCSK1 rs6234 with obesity and related traits in a Chinese Han population. Plos One. 2010;(5) Issue 5

12. Chang Y-C et al. Common variation in the fat mass and obesityassociated (FTO) gene confers risk of obesity and modulates BMI in the Chinese population. Diabetes. 2008; (57):2245-2252

13. Tan J et al. FTO variants are associated with obesity in the Chinese and Malay populations in Singapore. Diabetes. 2008; 57:8512857.:851-2857.

14. Hotta $\mathrm{K}$ et al. Variations in the FTO gene are associated with severe obesity in the Japanese. J Hum Genet. 2008; 53:546-553 
15. Herbert A et al. A common genetic variant is associated with adult and childhood obesity. Science. 2006; 312:279-283.

16. Hotta $\mathrm{K}$ et al. INSIG2 gene rs7566605 polymorphism is associated with severe obesity in Japanese. J Hum Genet. 2008; 53:857-862.

17. Cha $\mathrm{S}$ et al. Association analyses of the INSIG2 polymorphism in the obesity and cholesterol levels of Korean populations. BMC Medical Genetics. 2009;10:96.

18. Marvelle A, Lange L, Qin L, Adair L, and Mohlke K. Association of FTO with Obesity-Related Traits in the Cebu Longitudinal Health and Nutrition Survey (CLHNS) Cohort. Diabetes.2008 ; 57: 1987-1991.

19. Schrauwen P and Hesselink M. UCP2 and UCP3 in muscle controlling body metabolism. The Journal of Experimental Biology. 2002; 205: 2275-2285.

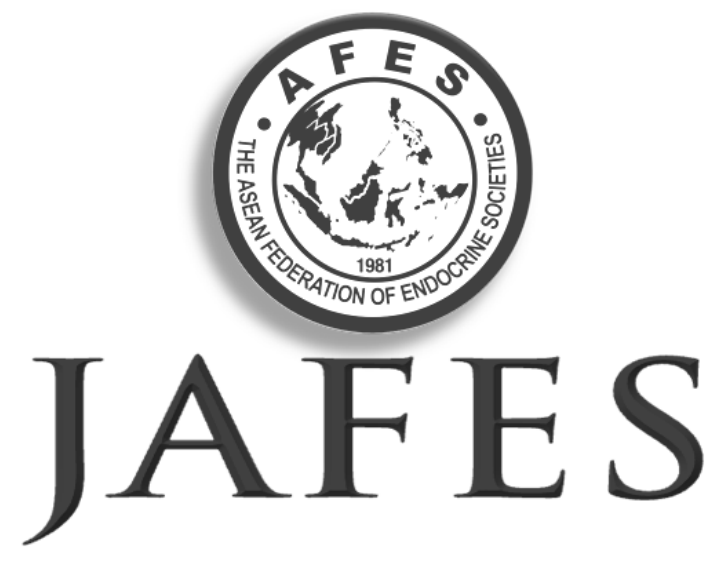

\section{Had an invigorating discussion in Grand Rounds? Share your Clinical Case Seminars at JAFES@Asia.com.}

